\title{
Arthroscopic Synovectomy in Rheumatoid Arthritis of Wrist
}

\author{
Sung-Jae Kim, MD, PhD and Kwang-Am Jung, MD
}

\begin{abstract}
The wrist is the most commonly involved region of the upper extremity in rheumatoid arthritis (RA). Because the wrist joint becomes involved early during the disease course and its involvement rapidly progresses, and because the disabilities associated with progressive RA are significant, early and adequate treatment must be introduced to prevent disease progression. Various treatment methods can be employed to treat RA wrists based on radiological and clinical findings. Arthroscopic synovectomy is recommended for pain relief and functional recovery in early stage RA, and is also helpful in advanced staged RA with Larsen stage III. However, arthroscopic synovectomy is not recommended as an effective method of treatment for all patients with advanced radiographic changes. Nevertheless, arthroscopic synovectomy may delay the need for complex surgery, such as wrist arthrodesis or total wrist arthroplasty in selective cases. Although arthroscopic synovectomy of the wrist cannot improve grip strength or range of motion, it can reduce wrist pain and improve function, and thus facilitate return to work.
\end{abstract}

Keywords: Arthroscopic synovectomy, Larsen stage III; Rheumatoid arthritis; Wrist

$\mathrm{T}$ he wrist is the most commonly involved region of the upper extremity in rheumatoid arthritis (RA), and about $75 \%$ of RA patients showed wrist symptoms. ${ }^{1,2}$ Because the wrist joint becomes involved early during the disease course and its involvement rapidly progresses, and because the disabilities associated with progressive RA are significant, early and adequate treatment must be introduced to prevent disease progression. RA of the wrist can be treated conservatively or surgically, such as synovectomy, wrist arthrodesis and arthroplasty. ${ }^{3-7}$ Conventional open surgical synovectomy is used to reduce symptoms and increases joint function in patients with early stage RA. In the early 1980s, arthroscopic synovectomy was first introduced to treat knee joints, and in the 1990s the technique and the instrumentation (particularly of small joint arthroscope and motorized instruments) were further developed and resulted in an increase in the use of wrist arthroscopy. ${ }^{8}$ Arthroscopic synovectomy produced results similar to those of conventional surgery and satisfactory results in RA patients with refractory wrist swelling.4,9-11 As compared with conventional surgical synovectomy, the arthroscopic technique has several advantages: less postoperative

Reprint Requests: Kwang-Am Jung, MD, Department of Orthopaedic Surgery, Himchan Hospital, 404-3, Mok-dong, Yangcheon-Ku, I 58-806 Seoul, KOREA, Tel: 822-3219-9।I4, Fax: 822-3219-9126,

Email: osjka@dreamwiz.com morbidity, minimal surgical trauma, short hospital stay and early rehabilitation. Arthroscopic synovectomy is commonly recommended in early stage RA, but it has not been recommended when a substantial degree of wrist joint destruction is present. In patients with advanced wrist damage, arthrodesis or wrist arthroplasty has been usually recommended. Recently, promising results for arthroscopic synovectomy have been reported in advanced staged RA of the wrist. ${ }^{4,10}$ Thus, we undertook to review published results of arthroscopic synovectomy for early stage and advanced stage RA of the wrist. $^{12}$

\section{ANATOMY AND PATHOMECHANISM}

The wrist provides stability and function to the hand. It is a complex structure comprised of three major synovial compartments: the radiocarpal, midcarpal and distal radioulnar, and surrounding stabilizing structures, such as the radiocarpal ligament, intercarpal ligament, triangular fibrocartilage complex (TFCC) and surrounding tendons. The distal radius and ulna articulate with each other at the distal radioulnar joint, and the distal radius articulates with the carpus (carpal bones) at the radiocarpal joint, which is

\author{
Received: April 2, 2007 \\ Revised: June 6, 2007 \\ Accepted: June 13, 2007
}

doi: $10.3|2| / \mathrm{cmr} .2007 .768$ 
essentially an articulation between the radius proximally and the scaphoid and lunate distally. The ulna is separated from the proximal row of carpal bones by the TFCC, a fibrocartilaginous disc that stretches from the medial aspect of the distal radius to the ulnar styloid. 2,13,14

Synovial hyperplasia with extensive infiltration by inflammatory cells is a characteristic of RA. Various studies have suggested that growth factors and cytokines like tumor necrosis factor (TNF) and interleukin (IL)-1 play important roles during the initiation and progression of RA. ${ }^{15-17}$ Moreover, because RA is essentially a synovial disease, each of the three separate compartments may become involved at disease onset. The tendon and ligament also become lax. In addition, when chronic rheumatoid joint inflammation persists, cartilage and periarticular bone are destroyed to variable extents due to the actions of inflammatory cytokines, and the resulting wrist imbalance may cause wrist deformity. Radiologically, periarticular osteoporosis, erosion, joint space narrowing, dislocation or ankylosis are typical findings in chronic RA. Radiocarpal involvement leads to rotatory instability of the scaphoid with resultant wrist collapse, loss of carpal height, ulnar and volar (palmar) translocation of the carpus on the radius, imbalance of the extensor tendon, a radial shift of metacarpals and ulnar deviation of fingers at metacarpophalangeal joints. The end stage rheumatoid wrist is dislocated volarly with complete destruction of the carpus and with dissociation of the distal radioular joint. ${ }^{13}$

\section{EVALUATION}

During early stage RA, wrist pain, tenderness and bulging of the dorsal wrist (which indicates synovitis) are developed and are reversible, whereas during advanced stage, prominence of ulnar head by ulnar dorsal subluxation of carpus and widening of the anterior-posterior dimension of the wrist by volar subluxation of carpus are likely. In addition, range of motion (flexion, extension, supination, pronation), grip strength and function may be affected, although standardized functional assessment methods did not seem to be. We routinely assess patients by allocating modified Mayo wrist scores (table 1). ${ }^{18}$ Standard radiographs play an important role during assessments of RA progression, and early diagnosis is required for proper management to prevent irreversible joint destruction, or at least retard disease progression. In recent study, magnetic resonance imaging (MRI) and ultrasound have enhanced usefulness. The study showed that the MRI revealed typical bony changes in early RA when conventional radiographs were normal. In addition, satisfactory results have been reported on the sensitivity and specificity of ultrasonography, especially for contrastenhanced ultrasonography for assessments of joint vascularity, a parameter of disease activity. ${ }^{19-26}$

\section{CLASSIFICATION}

In determining disease stage, assessments of erosion, joint space narrowing and cartilage loss by standard x-ray are considered an objective parameter. Many methods and

Table 1. The modified Mayo wrist scores.

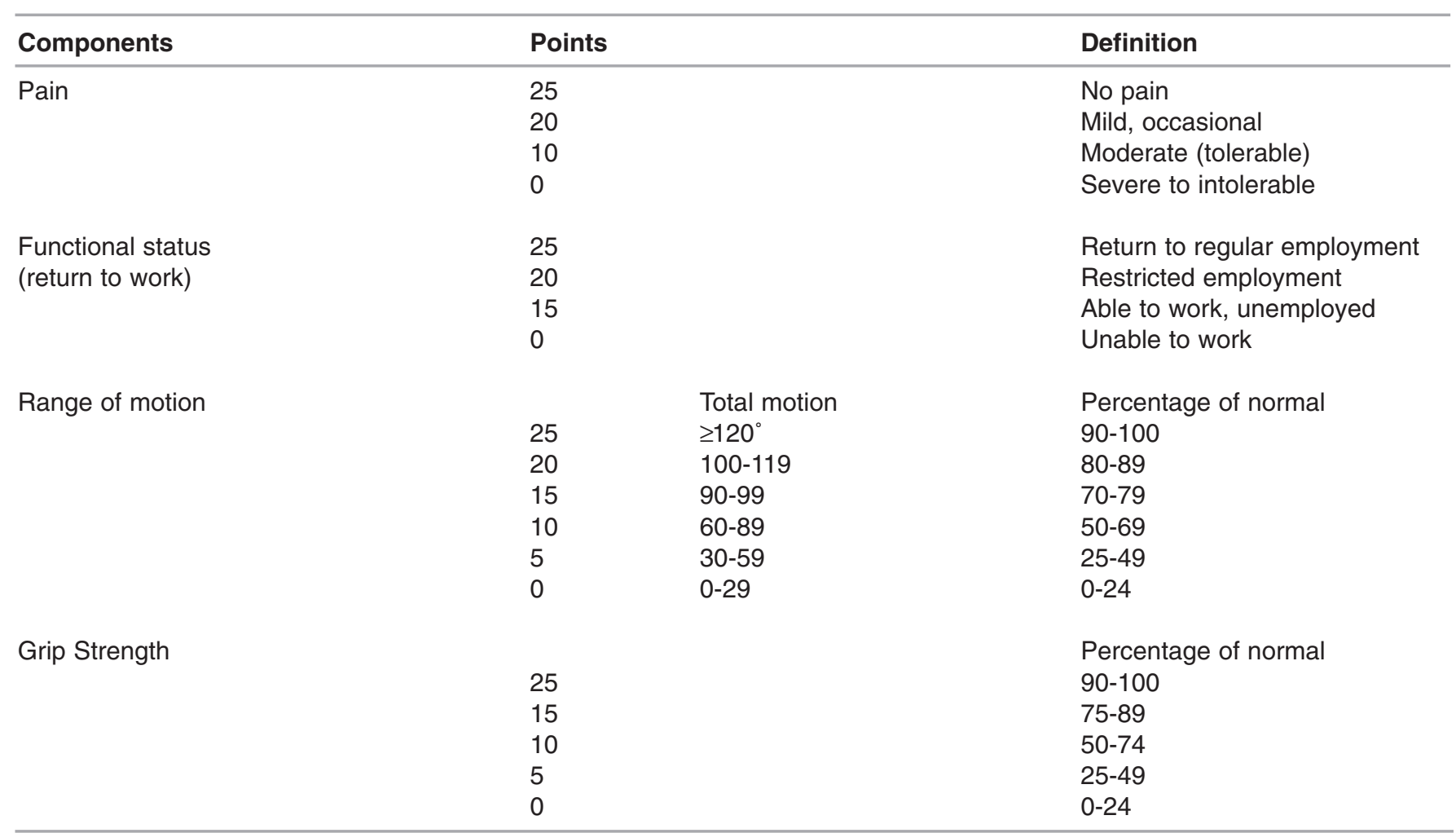

Excellent: 91-100 points; Good: 80-90 points; Fair: 65-79 points; Poor: $<65$ points. 
Table 2. Radiographic grading of rheumatoid arthritis according to Larsen et al.

\begin{tabular}{ll}
\hline Stage & Definition \\
\hline 0 & Normal conditions \\
1 & Soft tissue swelling, periarticular osteoporosis, and slight joint space narrowing \\
2 & Definite early abnormality, one or several small erosions \\
3 & Medium destructive abnormality, marked erosion \\
4 & Severe destructive abnormality, large erosion \\
5 & Mutilating abnormality (the original articular surfaces have disappeared), gross deformity \\
\hline
\end{tabular}

modifications of methods have been detailed since Larsen's radiological assessment was introduced in 1977 (table 2).2,10,27-29 These various assessment methods can be used to evaluate disease progression and determine treatment modalities adequately.

\section{TREATMENT}

The treatment of RA places a focus on pain relief and functional recovery, and on the resultant ability to work and improved life expectancy. Current therapeutic modalities cannot completely cure RA but do reduce discomfort and retard disease progression. The treatment is more efficient during early stage disease.

\section{Nonoperative Treatment}

A detailed understanding of the molecular immunology of RA has led to a transition from conventional treatments, such as nonsteroidal anti-inflammatory drugs (NSAID), glucocorticoids, disease-modifying anti-rheumatic drugs (DMARD) to aggressive immune suppression based on biological agents (TNF and IL-1 antagonists) that control TNF- $\alpha$ and IL-1, and thus, the inflammatory process. ${ }^{30} \mathrm{~A}$ brace is often used to maintain a stable joint position and in conjunction with modifying patient activity. 14,31,32 The introduction of biological agents has resulted in dramatic changes in the therapeutic approach and treatment paradigms for patients with RA. However, the cost should be considered because these agents are expensive. The National Institute for Health and Clinical Excellence (NICE) 2002 guidance for the use of TNF inhibitors recommended that etanercept and infliximab be used in patients with clinically active disease that has not responded adequately to at least two DMARDs, including methotrexate (unless contraindicated). There have been variable implementations of the guidelines with limited access to these agents in some areas. In the United States, the average wholesale price in 2006 for 1 year of therapy using the standard RA dose of either etanercept or adalimumab was approximately $\$ 16,000 .{ }^{33}$ Yen $^{34}$ recommended that a sufficient trial of conventional DMARDs should be performed before the use of expensive biological treatments in cases of early RA in developing countries. Some patients with chronic RA refractory to the above-described medical treatments also require local or surgical treatment. Medical synovectomy, which is an injection of chemicals or radioisotopes into the joints, has been used in clinical practice as a local treatment alternative to surgical synovectomy.
Chemical Synovectomy

The theoretical and practical advantages include minimal burden to the patient, simplicity of the technique with fewer associated risks, ability to reach areas inaccessible to surgery, minimal or no hospitalization, simple rehabilitation and low cost. Chemical synovectomy has historically been used with the help of various products, such as osmic acid, alkylating agents such as nitrogen mustard and thio-tepa, methotrexate and antibiotics. ${ }^{35}$ Eventually, nitrogen mustard, thio-tepa, methotrexate and antibiotics fell into disuse because of their adverse effects or lack of efficacy on the cartilage, even though osmic acid is still used occasionally in some countries. ${ }^{36-39}$

\section{RADiation SyNOVECTOMY}

Synovectomy by radioisotopes was first attempted in the 1950s. Radiation synovectomy is a regularly practiced nuclear medicine therapy in patients suffering from pain, recurrent joint effusion and restricted movement due to inflammatory and degenerative joint disease. ${ }^{40}$ The rationale of this technique is as follows: the radiopharmaceutical agent should have specific properties. The radiation energy should be sufficiently high to penetrate and ablate the synovial tissue but not so great as to damage the underlying articular cartilage or overlying skin. The radionuclide should be attached to particles that are sufficiently small to be phagocytosed but not so small that they might leak out of the joint before phagocytosis occurs. Furthermore, the particle should be biodegradable otherwise granulomatous tissue might form. Various $\beta$-emitting radioisotopes, e.g. Yttrium-90, Rhenium-186, erbium-169, are available. ${ }^{36,41}$ Following an intraarticular injection of a $\beta$-emitting radioisotope with a very short range, local irradiation resulted in sclerosis and finally fibrosis of the synovial membrane, achieving a significant reduction in pain and joint effusion. Radiation synovectomy is suitable for patients who suffer from clinical symptoms under standardized anti-rheumatoid drug therapy and in whom a pretherapeutic three-phase bone scan shows clear signs of hyperperfusion in their painful joints that is indicative of synovitis. The best results are produced in patients in the early stage of true RA. ${ }^{42-44}$ In RA wrist treated with radiation synovectomy using Rhenium-186, Kampen et al ${ }^{44}$ reported that the clinical symptoms showed improvement in 42 of 65 joints $(64.6 \%)$, which was supported by a decrease in post-therapeutic perfusion patterns in $59.1 \%$ at 6 - to 18 - months study. In addition, approximately $70 \%$ of patients treated for RA at various joints obtained good clinical results. 
Meanwhile, several studies reporting the results of arthroscopic synovectomy showed clinical improvement in almost all patients, even though the results of radiation synovectomy in the wrist joint could not be compared with surgical synovectomy because the tools for measuring the clinical and radiological outcomes in each study were not standardized. Radiation synovectomy has several advantages. It is free of surgical risk, includes the need for little or no hospitalization, does not involve physical therapy, has lower costs than the alternatives and allows repeated injection. Siegel et al ${ }^{45}$ reported that it also showed results equivalent to those of surgery. Therefore, radiation synovectomy appears to be an effective alternative to surgical synovectomy, and is also the procedure of choice for patients for whom surgery is contraindicated.

\section{Surgical Treatment}

In cases refractory to conservative treatment, surgical procedures are recommended. The generally accepted indications are when symptoms are not controlled despite conservative treatment for at least 6 to 9 months and when carpal deformity is found to progress. Various surgical procedures have been introduced with successful results, i.e., open synovectomy, arthroscopic synovectomy, wrist arthrodesis and total wrist arthroplasty. Choice of surgical procedure is based on radiologically-determined disease stage and also on the surgeon's preference. Open or arthroscopic synovectomy can provide pain relief with preserved or improved mobility in early stages of RA with joint space preservation, although synovectomy has been a controversial procedure.4,9,10 For patients with advanced radiographic changes, such as marked joint cartilage destruction, wrist arthrodesis or arthroplasty are usually recommended. Combined radiation and arthroscopic synovectomy also shows promise in treating RA.

Surgical synovectomy was first described more than 100 years ago and is a well accepted treatment method for RA joints. The theoretical rationale of synovectomy is to reduce or eliminate aggressive inflammatory cell mass, thereby improving function and reducing secondary pain.

\section{OPEN SYNOVECTOMY}

An open wrist synovectomy is standard and allows an inspection of all compartments as well as the extensor tendons. There is some controversy regarding the effectiveness of synovectomy. Several authors have reported that wrist synovectomy provides consistent and dramatic pain relief and maintains grip strength with a lower probability of tendon rupture, even if halting of the disease process is not demonstrated, but causes varying losses of wrist motion in the long term. ${ }^{13,40,46-50}$ However, some investigators claim that pain relief occurs at the expense of loss of joint motion and also the effect is ultimately transitory, with recurrence depending on the activity of the underlying cause of the arthritis. ${ }^{13}$ The wrist synovectomy is contraindicated in patients with rapidly progressed disease with joint destruction.
ARTHROSCOPIC SYNOVECTOMY

Although, an open wrist synovectomy is standard and allows an inspection of all compartments, as well as the extensor tendons, arthroscopic synovectomy has been used successfully in select patients. Arthroscopic synovectomy offers advantages over open tenosynovectomy. Arthroscopic synovectomy is a surgical procedure with minimal morbidity, which does not require open arthrotomy, and leaves less joint capsule and ligament damage, thus allowing immediate mobilization and reducing hospital stays. ${ }^{4,11,51}$ In addition, as compared with open arthrotomy, the arthroscopic technique offers superior views, easier access to radiocarpal and midcarpal compartments and facilitates the effective removal of pathologic synovium. ${ }^{52}$ Moreover, subsequent reductions in postoperative morbidity have made arthroscopic synovectomy widely applicable.

\section{Surgical Technique}

A standard wrist arthroscopy technique ${ }^{53,54}$ is performed with a patient placed in the supine position and the shoulder abducted at 90 degrees on an arm board. A pneumatic tourniquet is applied to the upper arm, and the forearm is suspended in a wrist traction device. The second and third fingers are then placed under 7 to 10 pounds of traction and a $1.9 \mathrm{~mm}$ diameter, 30 degree-arthroscope (Dyonics, Andover, MA) is initially placed into a 3 to 4 portal and then into a 6$\mathrm{R}$ portal for diagnostic wrist arthroscopy. Synovectomy of all visibly inflamed synovial tissues is performed using a $1.9 \mathrm{~mm}$ full-radius motorized suction shaving device, and debridement of pannus on the articular surface is then performed. To examine the midcarpal joint, another accessory portal is established, including the midcarpal radial and midcarpal ulnar portals. Debridement of the inflammatory synovial joints is done.

\section{Literature Review}

Arthroscopic synovectomy is a well-accepted method in early stage RA, but its use is controversial in advanced RA of the wrist. A search was made using the MESH terms, arthroscopic synovectomy, wrist and rheumatoid arthritis through the Medline and Embase databases and the Cochrane library for systematic reviews published from January 1977 to May 2007. The literature showed that arthroscopic synovectomies of rheumatoid wrists produced promising results. ${ }^{4,10}$ As compared with open synovectomy, the complications and morbidities associated with arthroscopic synovectomy are less. Adolfsson and Nylander ${ }^{9}$ reported the results of arthroscopic synovectomy in 18 wrists of 16 patients. Synovectomy was found to provide good pain relief in all cases, a motion increase from 69.5 to 90 degrees on average range of extension and flexion, and overall increase of grip strength of $87 \%$ in comparison with preoperative status at 6-month follow-ups. In another study by Adolfsson and Frisen ${ }^{4}$ with mean follow-up of 3.8 years, arthroscopic synovectomy was performed on 24 wrists with early stage RA. Pain was improved in 19 wrists, unchanged in 2 and worsened in 3. Range of motion increased from 74 to 90 
Table 3. Results using the modified Mayo wrist scores in unilateral wrists.

\begin{tabular}{lllllll}
\hline & \multicolumn{3}{c}{ Group A $(\mathbf{n = 1 1 )}$} & \multicolumn{3}{c}{ Group B (n=6) } \\
\hline & Preop & Follow-up & Increase & Preop & Follow-up & Increase \\
\hline Pain & 3.63 & $18.18^{*}$ & 14.54 & 6.25 & $20.00^{*}$ & 13.75 \\
$\begin{array}{l}\text { Range of motion } \\
\text { (MFEA) }\end{array}$ & $6.36\left(51.36^{\circ}\right)$ & $8.63\left(62.72^{\circ}\right)$ & 2.27 & $21.25\left(106.25^{\circ}\right)$ & $22.25\left(108.12^{\circ}\right)$ & 1.0 \\
Grip strength & 10.90 & 13.18 & 2.27 & & & \\
Functional status & 6.81 & $16.36^{*}$ & $9.55 \dagger$ & 20.62 & 21.87 & 1.25 \\
Total & 26.36 & $56.36^{*}$ & $30.5 \dagger$ & 67.5 & $83.75^{\star}$ & 1.25 \\
\hline
\end{tabular}

* Statistically significant difference $(P<0.05)$ at Mann Whitney $U$ test when follow-up compared with preop.

$\dagger$ Statistically significant difference $(P<0.05)$ at Mann Whitney $U$ test when increase in group A compared with increase in group B. Group A: Larsen stage III; Group B: Larsen stage I, II. Increase = follow-up - preop.

MFEA = mean flexion-extension arc.

Total score (points): Excellent: 91-100; Good: 80-90; Fair: 65-79; Poor <65.

degrees in the average flexion-extension arc and increased from 138 to 152 degrees in supination-pronation arc. In a study by Park et al 10 with a mean follow-up of 29 months, arthroscopic synovectomy was performed on 18 patients (19 wrists); preoperatively 13 wrists were radiologically Larsen stage I, 4 were Larsen stage II, 1 was Larsen stage III, and 1 was Larsen stage IV. Mean pain scores improved from 8 preoperatively to 4.5 postoperatively on the visual analogue scale (VAS; 10 points for worst possible pain and 0 points for no pain) and mean flexion-extension arc increased from 81 to 92 degrees. Patient satisfaction was high. The authors also recommend arthroscopic wrist synovectomy in patients with advanced radiographic changes and described promising results. However, their studies mainly focused on the outcome of arthroscopic synovectomy in patients with early-stage RA.4,9,10 Moreover, results of arthroscopic synovectomy for patients with Larsen stage III are not well described. In Adolfsson's review ${ }^{46}$ only a small number of patients with radiographic changes corresponding to grades III and IV of the Larsen-Dale-Eek index benefit from surgery and arthroscopic synovectomy was recommended on patients with stage II or less. However, advanced RA wrist results were not evaluated in detail. Although our previous study 12 was small in number, i.e., it included 11 patients with Larsen stage III RA with medium cartilage destruction (designated group A), this study revealed a promising clinical result for patients with Larsen stage III RA and medium cartilage destruction and joint space narrowing of $25 \%$ to $50 \%$. Referring to our previous study 12 in early stage RA (group B), pain was improved from 8 preoperatively to 4.5 postoperatively using the VAS scale. Assessments of individual components of modified Mayo wrist scores showed that pain scores increased from a mean of 6.25 preoperatively to 20.0 after an average follow-up of 69.5 months (range 33 to 118 months). Range of motion increased from 106 to 108 degrees in the average flexion-extension arc. However, no significant differences were observed between preoperative and postoperative score with respect to range of motion, grip strength and return to work status. Total modified Mayo wrist scores increased $(P=0.02)$ from an average of 67.5 points preoperatively to an average of 83.75 points at final follow-up.

Patients with Larsen stage III RA (group A) also showed improvements in pain score, which decreased from 8.4 preoperatively to 5.1 postoperatively in the VAS scale. The modified Mayo wrist score for pain increased from a mean of 3.63 preoperatively to 18.18 at final follow-up, return to work status score increased from 6.81 preoperatively to 16.36 at final follow-up and range of motion increased from 51 to 62 degrees. No significant differences in ROM or grip strength scores were observed. Total modified Mayo wrist scores increased from an average of 26.36 points preoperatively to an average of 56.36 points at final follow-up. On comparing groups A and B, group A was found to have a lower score for each preoperative component of the modified Mayo wrist scoring system than group B, with the exception of pain score. Increase was observed between preoperative and final follow-up scores in terms of return to work status and total modified Mayo wrist scores. Group A showed larger differences in this context than group B (table 3). Our data suggest that arthroscopic synovectomy is a useful treatment for modest and moderate RA and that it is particularly effective at reducing pain and improving function, and thus allowing patients to have early return to work. However, this does not mean that it prevents radiographic disease progression. In Adolfsson and Frisen's study ${ }^{4}$, which compared preoperative and postoperative $\mathrm{x}$-rays using the Larsen staging system, 10 of 13 wrists with grade II or III disease at the time of surgery revealed arthritic degenerative change progression by at least one grade, and this progression was significantly less common for preoperative grades 0 and I. In Park et al's study, ${ }^{10} 4$ of 19 wrists showed progression after synovectomy. These changes occurred slowly as reflected by mean modified Larsen scores ( 9.8 preoperatively and 13.9 at final follow-up). In our study, ${ }^{12}$ nine patients 
showed progression of RA to a higher radiographic grade, even though clinical symptoms improved. Postoperatively, 3 of 6 patients with Larsen stage I RA progressed to Larsen stage II RA, 1 of 2 patients with Larsen stage II RA progressed to Larsen stage IV RA, and 5 of 11 patients with Larsen stage III RA progressed to Larsen stage IV RA. Thus, synovectomy has not been shown to change natural disease progression, which did not seem to correlate with preoperative radiographic stage. Postoperatively, a team approach including the involvement of rheumatologists is recommended in the treatment plan.

\section{Complications}

Generally, arthroscopy of the wrist is a simple procedure without major complications. Park et $\mathrm{al}^{10}$ and Adolfsson et $\mathrm{al}^{4,9}$ reported no complications associated with surgery. In our study, ${ }^{12}$ one patient showed immediate numbness of the superficial radial nerve territory, which might have been injured at the 3 to 4 portal position but disappeared 1 month after surgery. More recently, injury to the posterior interosseous nerve has been reported during arthroscopy. ${ }^{55} \mathrm{In}$ order to avoid many potential complications, a blunt dissection to the level of the wrist capsule with a hemostat should be made to limit injury to the sensory nerves and extensor tendons. The instruments should not be forced into a joint because this increases the potential for iatrogenic chondral lesions. ${ }^{56}$

\section{COMBINED RADIATION AND ARTHROSCOPIC SYNOVECTOMY}

To our knowledge, there are no published articles on these combined procedures. Due to the anatomical characteristics of the wrist consisting of multiple articulations, a total synovectomy appears to be difficult to accomplish even though arthroscopy of the wrist has been advanced along with the development of instruments. Considering the abovedescribed rationale and the clinical result of both techniques, we believe that combined radiation and arthroscopic synovectomy can be performed to acquire a more complete synovectomy than arthroscopic synovectomy alone. However, an investigation into the clinical results and cost effectiveness of the combined procedure will be needed in the future.

\section{Wrist ARTHRODESIS AND TOTAL WRIST ARTHROPLASTY}

Wrist arthrodesis is the most commonly performed bony procedure in RA. Wrist fusion is indicated for advanced RA with severe radiocarpal instability, midcarpal involvement and the absence of wrist motors as a salvage procedure. ${ }^{2}$ However, a lack of patient satisfaction with fusion has led to continued efforts to develop a total wrist implant. The indication for total wrist arthroplasty is painful, pancarpal advanced RA in a patient willing to accept a low demand lifestyle, but who desires the ability to perform activities.

\section{SUMMARY}

Various treatment methods can be employed to treat RA wrists based on radiological and clinical situations. Arthroscopic synovectomy is recommended for pain relief and functional recovery in early stage RA, and also helpful in advanced staged RA with Larsen stage III. However, we cannot suggest that arthroscopic synovectomy is an effective method of treatment for all patients with advanced radiographic changes. Nevertheless, arthroscopic synovectomy may delay the need for complex surgery, such as wrist arthrodesis or total wrist arthroplasty, and also can be considered before performing arthrodesis or arthroplasty. Although arthroscopic synovectomy of the wrist cannot improve grip strength or range of motion, it can reduce wrist pain and improve function, and thus facilitate early return to work.

\section{ACKNOWLEDGMENTS}

The authors thank the Editor-in-Chief for inviting us to submit this article and Seok Won Kim for his assistance with the literature search.

\section{REFERENCES}

1. Flatt AE. The care of the arthritic hand. 5th ed. Saint Louis, MO: Quality Medical Publishing; 1995.

2. Ilan DI, Rettig ME. Rheumatoid arthritis of the wrist. Bull Hosp Jt Dis 2003;61:179-185.

3. Adams BD. Total wrist arthroplasty. Semin Arthroplasty. 2000;11:72-81.

4. Adolfsson L, Frisen M. Arthroscopic synovectomy of the rheumatoid wrist. A 3.8 year follow-up. J Hand Surg [Br] 1997;22:711-713.

5. Divelbiss BJ, Sollerman C, Adams BD. Early results of the Universal total wrist arthroplasty in rheumatoid arthritis. J Hand Surg [Am] 2002;27:195-204.

6. Kobus RJ, Turner RH. Wrist arthrodesis for treatment of rheumatoid arthritis. J Hand Surg [Am] 1990;15:541-546.

7. Millender LH, Nalebuff EA. Arthrodesis of the rheumatoid wrist. An evaluation of sixty patients and a description of a different surgical technique. J Bone Joint Surg Am 1973;55:1026-1034.

8. Roth JH, Poehling GG. Arthroscopic “-ectomy” surgery of the wrist. Arthroscopy 1990;6:141-147.

9. Adolfsson L, Nylander G. Arthroscopic synovectomy of the rheumatoid wrist. J Hand Surg [Br] 1993;18:92-96.

10. Park MJ, Ahn JH, Kang JS. Arthroscopic synovectomy of the wrist in rheumatoid arthritis. J Bone Joint Surg Br 2003;85:1011-1015.

11. Wei N, Delauter SK, Beard S, Erlichman MS, Henry D. Officebased arthroscopic synovectomy of the wrist in rheumatoid arthritis. Arthroscopy 2001;17:884-887.

12. Kim SJ, Jung KA, Kim JM, Kwun JD, Kang HJ. Arthroscopic synovectomy in wrists with advanced rheumatoid arthritis. Clin Orthop Relat Res 2006;449:262-266.

13. Rosen A, Weiland AJ. Rheumatoid arthritis of the wrist and hand. Rheum Dis Clin North Am 1998;24:101-128.

14. Blank JE, Cassidy C. The distal radioulnar joint in rheumatoid arthritis. Hand Clin 1996;12:499-513.

15. Arend WP, Dayer JM. Inhibition of the production and effects of interleukin-1 and tumor necrosis factor alpha in rheumatoid arthritis. Arthritis Rheum 1995;38:151-160.

16. Eastgate JA, Symons JA, Wood NC, Grinlinton FM, di Giovine FS, Duff GW. Correlation of plasma interleukin 1 levels with disease activity in rheumatoid arthritis. Lancet 1988;2: 706-709.

17. Kahle P, Saal JG, Schaudt K, Zacher J, Fritz P, Pawelec G. Determination of cytokines in synovial fluids: correlation with diagnosis and histomorphological characteristics of synovial tissue. Ann Rheum Dis 1992;51:731-734. 
18. Cooney WP, Linscheid RL, Dobyns JH. Triangular fibrocartilage tears. J Hand Surg [Am] 1994;19:143-154.

19. Backhaus M, Kamradt T, Sandrock D, Loreck D, Fritz J, Wolf KJ, Raber H, Hamm B, Burmester GR, Bollow M. Arthritis of the finger joints: a comprehensive approach comparing conventional radiography, scintigraphy, ultrasound, and contrast-enhanced magnetic resonance imaging. Arthritis Rheum 1999;42:1232-1245.

20. Boutry N, Larde A, Lapegue F, Solau-Gervais E, Flipo RM, Cotten A. Magnetic resonance imaging appearance of the hands and feet in patients with early rheumatoid arthritis. J Rheumatol 2003;30:671-679.

21. McQueen FM. Magnetic resonance imaging in early inflammatory arthritis: what is its role? Rheumatology (Oxford) 2000;39:700-706.

22. Peterfy CG. Magnetic resonance imaging in rheumatoid arthritis: current status and future directions. J Rheumatol 2001;28:1134-1142.

23. Scott DL, Coulton BL, Popert AJ. Long term progression of joint damage in rheumatoid arthritis. Ann Rheum Dis 1986;45:373-378.

24. Sugimoto H, Takeda A, Hyodoh K. Early-stage rheumatoid arthritis: prospective study of the effectiveness of MR imaging for diagnosis. Radiology 2000;216:569-575.

25. Sugimoto H, Takeda A, Masuyama J, Furuse M. Early-stage rheumatoid arthritis: diagnostic accuracy of MR imaging. Radiology 1996;198:185-192.

26. Wieners G, Detert J, Streitparth F, Pech M, Fischbach F, Burmester G, Ricke J, Backhaus M, Bruhn H. Highresolution MRI of the wrist and finger joints in patients with rheumatoid arthritis: comparison of 1.5 Tesla and 3.0 Tesla. Eur Radiol 2007;17:2176-2182.

27. Hodgson SP, Stanley JK, Muirhead A. The Wrightington classification of rheumatoid wrist $\mathrm{X}$-rays: a guide to surgical management. J Hand Surg [Br] 1989;14:451-455.

28. Larsen A, Dale K, Eek M. Radiographic evaluation of rheumatoid arthritis and related conditions by standard reference films. Acta Radiol Diagn (Stockh) 1977;18: 481-491.

29. Zangger P, Kachura JR, Bogoch ER. The Simmen classification of wrist destruction in rheumatoid arthritis. Experience in patients with early disease. J Hand Surg [Br] 1999;24: 400-404.

30. Howe CR, Gardner GC, Kadel NJ. Perioperative medication management for the patient with rheumatoid arthritis. J Am Acad Orthop Surg 2006;14:544-551.

31. Someya F, Miaki H, Asai H, Tachino K, Nara I. Hand splint for rheumatoid arthritis patients during gait training after joint replacement in lower extremity. Arch Phys Med Rehabil 1988;69:644

32. Spoorenberg A, Boers M, Van der Linden S. Wrist splints in rheumatoid arthritis: a question of belief? Clin Rheumatol 1994;13:559-563.

33. Kavanaugh A. Economic issues with new rheumatologic therapeutics. Curr Opin Rheumatol 2007;19:272-276.

34. Yen JH. Treatment of early rheumatoid arthritis in developing countries. Biologics or disease-modifying anti-rheumatic drugs? Biomed Pharmacother 2006;60:688-692.

35. Cruz-Esteban C, Wilke WS. Innovative treatment approaches for rheumatoid arthritis. Non-surgical synovectomy. Baillieres Clin Rheumatol 1995;9:787-801.

36. Heuft-Dorenbosch LL, de Vet HC, van der Linden S. Yttrium radiosynoviorthesis in the treatment of knee arthritis in rheumatoid arthritis: a systematic review. Ann Rheum Dis 2000;59:583-586.

37. Markovits D, Rozenbaum M, Rosner I, Rottenstriech E. Chemical synovectomy in arthritis by intra-articular injection of osmic acid. Harefuah 1998;134:520-523, 591.
38. Gristina AG, Pace NA, Kantor TG, Thompson WA. Intraarticular thio-tepa compared with depomedrol and procaine in the treatment of arthritis. J Bone Joint Surg Am 1970;52:1603-1610.

39. Menkes CJ. Is there a place for chemical and radiation synovectomy in rheumatic diseases? Rheumatol Rehabil 1979;18:65-77.

40. Thirupathi RG, Ferlic DC, Clayton ML. Dorsal wrist synovectomy in rheumatoid arthritis - a long-term study. J Hand Surg [Am] 1983;8:848-856.

41. Muller W, Pavelka K, Fridrich R. Treatment of chronic articular effusions with 90-yttrium (90Y). Scand J Rheumatol 1975;4:216-220.

42. Menkes CJ, Go AL, Verrier P, Aignan M, Delbarre F. Doubleblind study of erbium 169 injection (synoviorthesis) in rheumatoid digital joints. Ann Rheum Dis 1977;36:254-256

43. Boussina I, Toussaint M, Ott H, Hermans P, Fallet GH. A double-blind study of erbium 169 synoviorthesis in rheumatoid digital joints. Results after one year. Scand J Rheumatol 1979;8:71-74.

44. Kampen WU, Brenner W, Kroeger S, Sawula JA, Bohuslavizki $\mathrm{KH}$, Henze E. Long-term results of radiation synovectomy: a clinical follow-up study. Nucl Med Commun 2001; 22:239-246.

45. Siegel ME, Siegel HJ, Luck JV Jr. Radiosynovectomy's clinical applications and cost effectiveness: a review. Semin Nucl Med 1997;27:364-371.

46. Adolfsson L. Arthroscopic synovectomy in wrist arthritis. Hand Clin 2005;21:527-530.

47. Allieu Y, Lussiez B, Ascencio G. Long-term results of surgical synovectomies of the rheumatoid wrist. Apropos of 60 cases. Rev Chir Orthop Reparatrice Appar Mot 1989;75:172-178.

48. Brumfield R Jr, Kuschner SH, Gellman H, Liles DN, Van Winckle G. Results of dorsal wrist synovectomies in the rheumatoid hand. J Hand Surg [Am] 1990;15:733-735.

49. Canale ST. Campbell's operative orthopaedics. 10th Ed. St Louis, MO: Mosby; 2003. 3729-3734.

50.Shapiro JS, Rodts T, Labanauskas I, Payne T. Early synovectomy versus late arthroplasty of the rheumatoid wrist. A comparative study. J Hand Surg 1985;10A:430.

51. Sculco TP. The knee joint in rheumatoid arthritis. Rheum Dis Clin North Am 1998;24:143-156.

52. Osterman AL. Wrist arthroscopy: Operative procedures. In: Green DP, Hotchkiss RN, Pederson WC, eds. Green's operative hand surgery. 4th ed. Philadelphia, PA: Churchill Livingstone; 1999. 207-222.

53. Abrams RA, Petersen M, Botte MJ. Arthroscopic portals of the wrist: an anatomic study. J Hand Surg [Am] 1994;19:940-944.

54. Whipple TL, Marotta JJ, Powell JH 3rd. Techniques of wrist arthroscopy. Arthroscopy 1986;2:244-252.

55. del Pinal F, Herrero F, Cruz-Camara A, San Jose J. Complete avulsion of the distal posterior interosseous nerve during wrist arthroscopy: a possible cause of persistent pain after arthroscopy. J Hand Surg [Am] 1999;24:240-242.

56. Gupta R, Bozentka DJ, Osterman AL. Wrist arthroscopy: principles and clinical applications. J Am Acad Orthop Surg 2001;9:200-209.

\section{AUTHOR AFFILIATIONS}

Sung-Jae Kim, MD, PhD

Department of Orthopaedic Surgery

Yonsei University College of Medicine

Seoul, Korea

Kwang-Am Jung, MD

Department of Orthopaedic Surgery

Himchan Hospital

Seoul, Korea 